\title{
Stator Winding Fault Phase Identification Using Piezoelectric Sensors in Three-Phase Induction Motors ${ }^{+}$
}

\author{
Guilherme Lucas* ${ }^{\mathbb{D}}$, Marco Rocha $\mathbb{D}^{\mathbb{D}}$, Bruno Castro $\mathbb{D}^{\mathbb{B}}$, José Leão and André Andreoli ${ }^{\mathbb{D}}$ \\ Department of Electrical Engineering, São Paulo State University (UNESP), Bauru 17033-360, Brazil \\ * Correspondence: guilherme.beraldi@unesp.br \\ + Presented at the 7th Electronic Conference on Sensors and Applications, 15-30 November 2020; \\ Available online: https:/ /ecsa-7.sciforum.net/.
}

Published: 15 November 2020

\begin{abstract}
Three Phase Induction Motors (TIMs) play a key role in industrial production lines. Due to its robustness and versatility, TIMs are commonly used to drive different devices like fans, conveyors, sieves, and compressors. However, these equipment are often exposed to mechanical and electrical faults. Among them, failures in stator winding insulation lead to severe damage to the TIMs and can cause operational interruptions. Therefore, several approaches have been developed to monitor electrical faults in induction motors. The acoustic emission (AE) stands out as an efficient non-invasive technique (NIT) for TIM diagnosis. In this work, the AE analysis was applied to detect winding insulation faults and identify which electrical phase was affected. To achieve this proposal, a TIM was subjected to insulation faults in each of the three electrical phases, and the acoustic signals were acquired by four piezoelectric sensors attached to the motor. These signals were processed using a new technique, which calculates the energy of a specific range of the signal spectrum and assigns the energy values of each piezoelectric sensor to a coordinate axis $(x, y)$. By ploting the values for each fault condition, this technique allows the detection of insulation faults and correctly identifies the affected phase by clustering the resulting values. Finally, the proposed methodology presented satisfactory results in winding insulation diagnosing.
\end{abstract}

Keywords: winding fault; acoustic emission; piezoelectric sensors; induction motors

\section{Introduction}

Three-phase induction motors (TIMs) are robust, durable, and efficient machines that are widely applied to drive different types of industrial loads. Hence, any unplanned interruptions of its operation are directly related to productive and financial losses [1]. Therefore, monitoring and diagnosis techniques are growing trends in the modern industrial scenario. As a consequence, sensor-based systems have been massively developed, focusing on TIMs fault detection and identification [2,3]. According to [4], stator failures are the second most common type of motor failures and can lead to severe damage to the TIM. Among them, inter-turn short circuits (ITSCs) frequently affects induction motors. ITSCs generate overcurrent, winding overheating, torque losses, and insulation deterioration [5]. Therefore, several monitoring techniques have been proposed to diagnose this fault. However, non-invasive techniques (NIT) stand out for their ability to perform TIM monitoring without interrupting the regular machine operation [6].

In this context, this work proposes an ITSC detection and phase identification system by using acoustic emission (AE) sensors, which consists of an NIT for TIM diagnosing. To achieve this goal, the three phases of a TIM were individually submitted to inter-turn short circuits. Also, during the 
application of ITSCs, the acoustic signals of four piezoelectric sensors were acquired. After that, these signals were processed using a new grouping technique, which calculates the signal energy in a specific range of the frequency domain. The clustering behavior was achieved by assigning these energy values from two different sensors to the $x$ and $y$ coordinated axis, respectively. This processing technique allows a novel acoustic emission diagnosis that detects the occurrence of the short circuit and identifies which phase was affected.

In conclusion, based on the relevance of the TIMs, it is crucial to develop fault detection methods for this equipment [7]. Also, the acoustic-based technique proposed in this work proved to be an efficient NIT for ITSC diagnosing. The piezoelectric sensors applied to signal acquisition are considerably cheaper than conventional AE sensors, allowing the development of viable monitoring systems. Finally, the results showed that the novel clustering method was able to correctly diagnose the ITSC condition.

This paper is divided into six sections. Sections 2 and 3 describe the piezoelectric sensors and the signal processing technique, respectively. Section 4 describes the experimental setup that was carried out in this work. Section 5 contains the results, and Section 6 presents the conclusions.

\section{Acoustic Sensors}

The piezoelectric effect is defined as a reversible electromechanical iteration that can be observed in some specific materials. This property allows the piezoelectric materials to act as an acoustic transducer, i.e., it can convert the acoustic emissions into electrical signals [8]. Consequently, several studies have taken advantage of this property, including fault detection and structure monitoring $[9,10]$.

The piezoelectric sensors applied in this work were the piezoelectric diaphragms. Their basic construction consists of a thin piezoelectric ceramic disk covered by a silver electrode and positioned over a circular brass electrode (Figure 1). The circular base dimensions are $20 \mathrm{~mm} \times 0.2 \mathrm{~mm}$ and the circular piezoelectric ceramic dimensions are $14 \mathrm{~mm} \times 0.42 \mathrm{~mm}$. Finally, the AE signals provided by the piezoelectric diaphragms were processed and analyzed using the new technique based on Fast Fourier Transform (FFT), which will be described in the next Section.

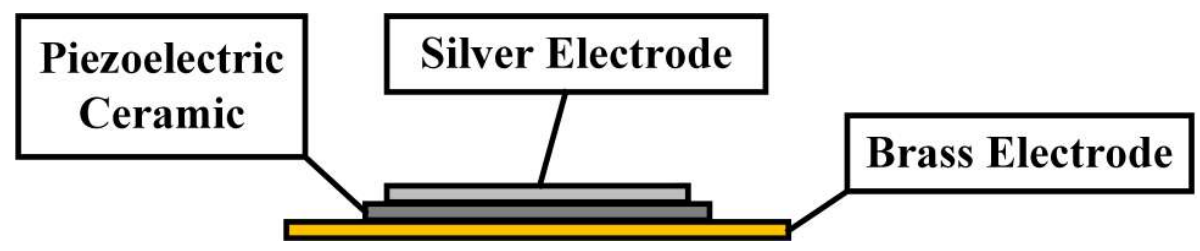

Figure 1. Piezoelectric sensor representation.

\section{Acoustic Signals Analysis}

The novel NIT developed in this article is based on the Signal Energy and the Fast Fourier Transform. The following subsections will present and formulate both of these algorithms used to process and analyze the acquired data.

\subsection{Fast Fourier Transform}

The algorithm used to transform a digital signal in the time domain into its representation in frequency domain is known as Discrete Fourier Transform (DFT). For a digital signal $x(n)$, with $n=0$, $1,2, \ldots, N-1$, and the twiddle factors $\omega_{N}$ associated to it are defined by Equation (1) [11]:

$$
X(k)=\sum_{n=0}^{N-1} x(n) \omega_{N}^{k n}
$$

Due to computational effort, it was used the Fast Fourier Transform (FFT) to calculate the DFT coefficients [11]. 
Due to capacity to evaluate the frequency behavior of signals, FFT analysis has been used several times in the diagnosis of electrical machines [12,13]. Therefore, the proposed technique uses Fast Fourier Analysis to perform a diagnose of inter-turn short circuit fault in stator of induction machine.

\subsection{Signal Energy}

The energy of acoustic signals is a well known metric for fault diagnosis. In TIM monitoring, AE sensors are applied to detect variations in the energy of acoustic signals $[14,15]$. Atypical variations usually indicate an anomaly. Therefore, the acoustic energy was used in this work as a index to assess the ITSCs.

The energy value can be describe as Equation (2) [14]:

$$
E=\sum_{j=1}^{N}\left|X_{j}\right|^{2}
$$

where $N$ is the length of signal $X$.

\section{Experimental Setup}

The objective of this work is to apply the acoustic piezoelectric sensors in the inter-turn short circuit fault diagnosis. The procedure for the acquisition of AE signals and the experimental setup of electrical machines are described in the following subsections.

\subsection{Test Bench Setup}

The test bench was assembled using a $1.5 \mathrm{hp}$ TIM coupled to a DC machine. Both of them were aligned and firmly bolted. The three-phase induction motor is powered at nominal voltage by a Pacific Power 360 AMX source, and the DC machine was used as a generator to supply restive loads, providing nominal torque on the TIM shaft. The three-phase induction motor was specifically designed to allow access to stator windings, as shown in the Figure 2a. In order to emulate an ITSC in the TIM, a $10 \Omega$ resistor was connected between the winding sections 1 and 3 , as shown in Figure $2 \mathrm{~b}$. This procedure corresponds to a $11 \%$ short circuit regarding the entire winding and was repeated for the three phases. To improve the reliability of the diagnosis, a baseline experiment was performed in nominal operation. Also, each experiment was repeated 50 times to ensure the repeatability of the experiments.

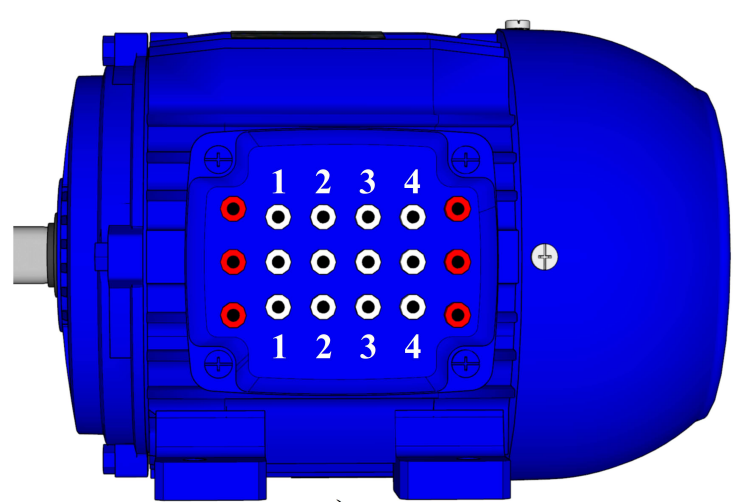

a)

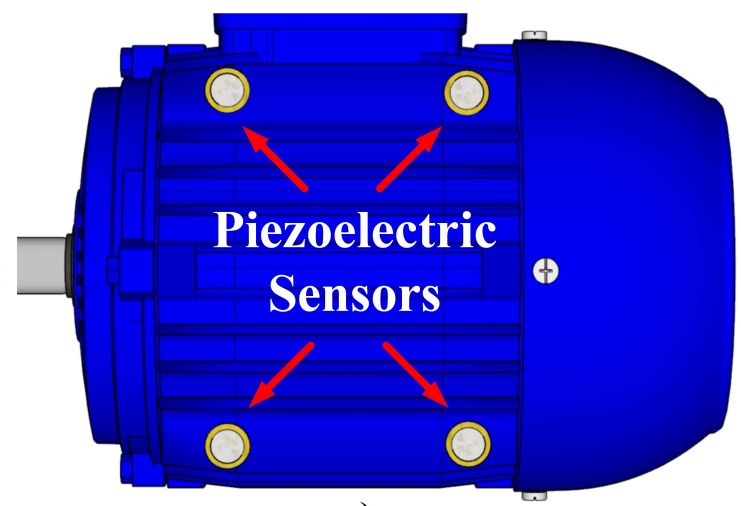

c)

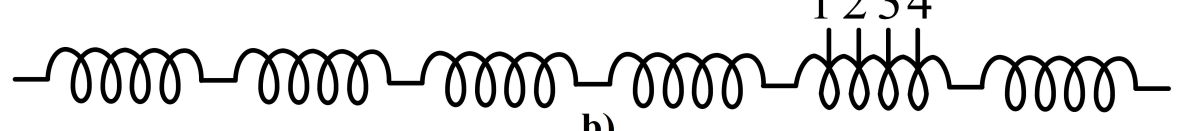

b)

Figure 2. (a) Winding external connections. (b) Winding internal sections. (c) Piezoelectric sensors positions. 


\subsection{Sensor and Data Acquisition}

To accomplish the signal acquisition, four acoustic piezoelectric sensors were attached to the TIM frame (Figure 2c) during the ITSC experiments. The AE signals were acquired by a digital oscilloscope with a sample rate of $2 \mathrm{MS} / \mathrm{S}$, which matches the Nyquist theorem. Also, a conditioning board was used as a anti-aliasing filter. Finally, the data were processed using a personal computer where the FFT and Energy values were calculated and the results were analyzed using the software MATLAB ${ }^{\circledR}$.

\section{Results}

After the application of FFT to AE signals, the spectra of the signals acquired under healthy condition (baseline) was compared to the spectra of the signals acquired under ITSC condition. The comparison showed amplitude variations between healthy and fault situations in the frequency band starting at 800 to $850 \mathrm{~Hz}$, as shown in Figure 3. Also, it was noted that each sensor presented a different response depending on affected phase or baseline. It can be explained by their geometric positions and the internal winding distribution. This feature allowed the development of a new clustering technique based on the different energy values contained within this band for each sensor. The technique consists in assigning the energy values of two sensors to the $\mathrm{x}$ and $\mathrm{y}$ axes of a Cartesian Plane. Three different combinations of sensors were tested and the results can be seen in Figure 4.

By analyzing Figure 4, it was observed that the resulting clusters presented a remarkable distancing between each affected phase and the baseline. Also, all the combinations presented good clustering results. Additionally, it was implemented the k-means algorithm to determine the centroids, that are also presented in the Figure 4. Using the centroids it was possible to calculate the Silhouette and Precision indexes. The first one analyzes the accuracy of the clusters by measuring how close a point is to the other points of the same cluster. The last one evaluates the precision of the technique by verifying if all points were correctly related to each affected phase or baseline. The Silhouette index is measured from -1 (low accuracy) to 1 (high accuracy) and the Precision values are measured from 0 (low precision) to 1 (high precision). The Silhouette indexes related to the three combinations (sensor 4 and 2-sensor 4 and 1-sensor 4 and 3) are, respectively: 0.95, 0.97 and 0,94, proving the high accuracy of the novel technique. Similarly, all combinations reached the maximum Precision value, as can be seen in Figure 4.

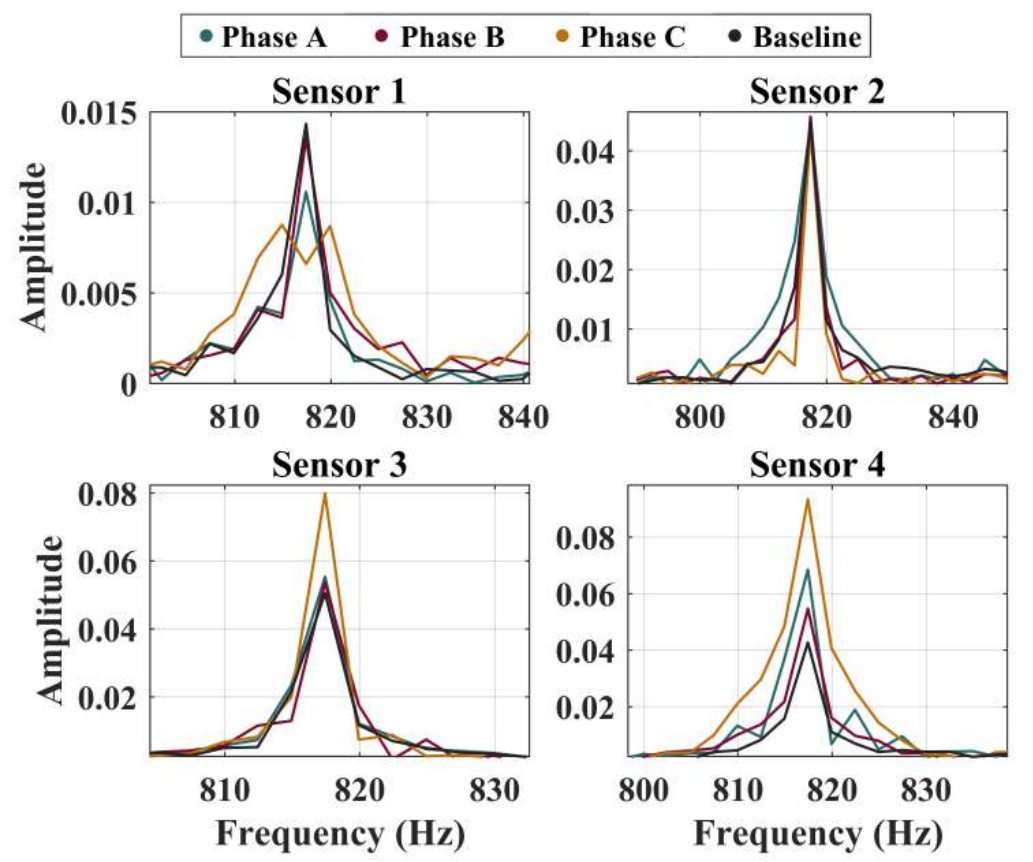

Figure 3. Spectral comparison between baseline and fault situations for each phase. 


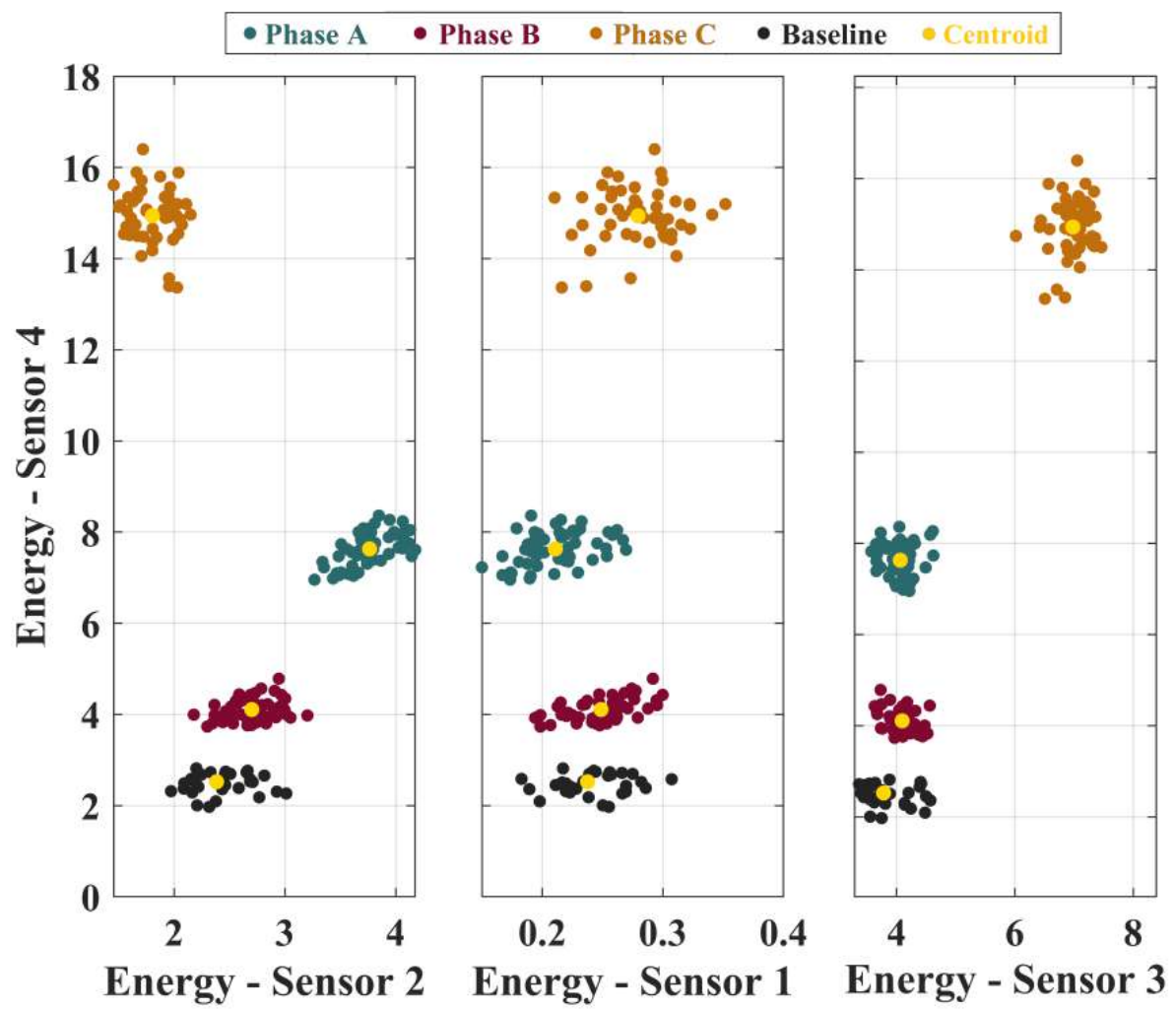

Figure 4. Phase identification clusters for ITSC.

\section{Conclusions}

Due to the relevance of TIM in production lines and industrial environment, reliable diagnosis systems are powerful tools to improve the maintenance plans. Therefore, in this work, it was developed a NIT that uses a low-cost acoustic sensor to perform a detailed analysis of inter-turn short circuits in these machines.

The application of the new technique has stood out for providing precise and high accuracy results, as proved by Silhouette and Precision indexes. It allowed to correctly detect the occurrence of a ITSC and also identify which phase was affected by this condition.

In future works, more than one phase can be affected by ITSC at the same time. Also, it can be proposed the classification of different levels of ITSC using the same signal processing technique.

Funding: This research was funded by Coordination of Superior Level Staff Improvement Personnel (grant \#88882.432803/2019-01).

Conflicts of Interest: The authors declare no conflict of interest.

\section{References}

1. Gangsar, P.; Tiwari, R. A support vector machine based fault diagnostics of Induction motors for practical situation of multi-sensor limited data case. Measurement 2019, 135, 694-711, doi:10.1016/j.measurement.2018.12.011.

2. Jiang, C.; Li, S.; Habetler, T.G. A review of condition monitoring of induction motors based on stray flux. In Proceedings of the 2017 IEEE Energy Conversion Congress and Exposition (ECCE), Cincinnati, OH, USA, 1-5 October 2017. doi:10.1109/ecce.2017.8096907.

3. Chen, J.; Hu, N.; Zhang, L.; Chen, L.; Wang, B.; Zhou, Y. A Method for Broken Rotor Bars Diagnosis Based on Sum-Of-Squares of Current Signals. Appl. Sci. 2020, 10, 5980, doi:10.3390/app10175980.

4. Dorrell, D.G.; Makhoba, K. Detection of Inter-Turn Stator Faults in Induction Motors Using Short-Term Averaging of Forward and Backward Rotating Stator Current Phasors for Fast Prognostics. IEEE Trans. Magn. 2017, 53, 1-7, doi:10.1109/tmag.2017.2710181,. 
5. Xu, Z.; Hu, C.; Yang, F.; Kuo, S.H.; Goh, C.K.; Gupta, A.; Nadarajan, S. Data-Driven Inter-Turn Short Circuit Fault Detection in Induction Machines. IEEE Access 2017, 5, 25055-25068, doi:10.1109/access.2017.2764474.

6. Gangsar, P.; Tiwari, R. Signal based condition monitoring techniques for fault detection and diagnosis of induction motors: A state-of-the-art review. Mech. Syst. Signal Process. 2020, 144, 106908, doi:10.1016/j.ymssp.2020.106908.

7. Ali, M.Z.; Shabbir, M.N.S.K.; Liang, X.; Zhang, Y.; Hu, T. Machine Learning-Based Fault Diagnosis for Singleand Multi-Faults in Induction Motors Using Measured Stator Currents and Vibration Signals. IEEE Trans. Ind. Appl. 2019, 55, 2378-2391, doi:10.1109/tia.2019.2895797.

8. Fraden, J. Handbook of Modern Sensors : Physics, Designs, and Applications; Springer: Cham, Switzerland; New York, NY, USA, 2016.

9. Carvalho, L.; Lucas, G.; Rocha, M.; Fraga, C.; Andreoli, A. Undervoltage Identification in Three Phase Induction Motor Using Low-Cost Piezoelectric Sensors and STFT Technique. Proceedings 2019, 42, 72, doi:10.3390/ecsa-6-06644.

10. Zhen, D.; Wang, Z.; Li, H.; Zhang, H.; Yang, J.; Gu, F. An Improved Cyclic Modulation Spectral Analysis Based on the CWT and Its Application on Broken Rotor Bar Fault Diagnosis for Induction Motors. Appl. Sci. 2019, 9, 3902, doi:10.3390/app9183902.

11. Proakis, J. Digital Signal Processing; Pearson Prentice Hall: Upper Saddle River, NJ, USA, 2007.

12. Ewert, P.; Kowalski, C.T.; Orlowska-Kowalska, T. Low-Cost Monitoring and Diagnosis System for Rolling Bearing Faults of the Induction Motor Based on Neural Network Approach. Electronics 2020, 9, 1334, doi:10.3390/electronics9091334.

13. Gonzalez-Toral, R.; Reviriego, P.; Maestro, J.A.; Gao, Z. A Scheme to Design Concurrent Error Detection Techniques for the Fast Fourier Transform Implemented in SRAM-Based FPGAs. IEEE Trans. Comput. 2018, 67, 1039-1045, doi:10.1109/tc.2018.2792445.

14. Lucas, G.B.; de Castro, B.A.; Rocha, M.A.; Andreoli, A.L. Three-phase induction motor loading estimation based on Wavelet Transform and low-cost piezoelectric sensors. Measurement 2020, 164, 107956, doi:10.1016/j.measurement.2020.107956.

15. Zhao, M.; Xu, G. Feature extraction of power transformer vibration signals based on empirical wavelet transform and multiscale entropy. IET Sci. Meas. Technol. 2018, 12, 63-71, doi:10.1049/iet-smt.2017.0188.

(C) 2020 by the authors. Licensee MDPI, Basel, Switzerland. This article is an open access article distributed under the terms and conditions of the Creative Commons Attribution (CC BY) license (http:/ / creativecommons.org/licenses/by/4.0/). 